Vol. 43 (1991) [325-329]

\title{
ON THE GENERATORS OF $S$-UNIT GROUPS IN ALGEBRAIC NUMBER FIELDS
}

\author{
B. BRINDZA
}

\begin{abstract}
Given a finitely generated multiplicative subgroup $U_{S}$ in a number field, we employ a simple argument from the geometry of numbers and an inequality on multiplicative dependence in number fields to obtain a minimal set of generators consisting of elements of relatively small height.
\end{abstract}

\section{INTRODUCTION}

Let $K$ be an algebraic number of degree $n$ over $Q$, with discriminant $D$, regulator $R$ and class number $h$. Let $r$ denote the rank of its group of units.

Denote by $S$ a finite set of absolute values of $K$ including all its archimedean (infinite) values; let $s$ be its cardinality. An element $\alpha$ of $\mathrm{K}$ is called an $S$-unit if $|\alpha|_{v}=1$ for every absolute value not in $S$. It is a well known generalisation of Dirichlet's unit theorem that the $S$-units of $K$ form a finitely generated subgroup $U_{S}$ of rank $s-1$ in $K^{x}$.

It turns out that wide classes of diophantine problems can be reduced to additive relations on $S$-units. It is therefore of interest to find effective bounds for the generators of groups $U_{S}$. Of course, it is a simple matter to construct $s-1$ multiplicatively independent elements by using the prime ideals corresponding to the nonarchimedean values in $S$. That yields a subgroup of finite index in $U_{S}$ (see, for example [3], Lemma 4). However, the best known bounds for the "size" of the representatives of the quotient group is exponential in $n$ and $R$.

In this note we construct a set of generators $\pi_{1}, \ldots, \pi_{1-1}$ for the non-torsion subgroup of $U_{S}$ (so that its quotient with $U_{S}$ is just the cyclic group of roots of unity in $K)$.

Received 16 May 1990

Work supported by an Australian Research Council National Research Fellowship held at Macquarie University

Copyright Clearance Centre, Inc. Serial-fee code: 0004-9729/91 \$A2.00+0.00. 


\section{The Main Result}

As usual we denote by $h()$ the absolute logarithmic height

$$
h(\gamma)=\frac{1}{[K: Q]} \log \left(\prod_{v} \max \left(1,|\gamma|_{v}\right)\right)
$$

of elements of $K$ (with the product running over the values $v$ of $K$, so normalised that one has the product formula and that rational integers $h$ have $\mathrm{h}(h)=\log h)$. Furthermore, let $\mathfrak{p}_{1}, \ldots, \mathfrak{p}_{t}$ be the prime ideals of $K$ corresponding to the nonarchimedean (finite) values of $S$ and set $P=\max _{j}\left(2, \operatorname{Norm} \mathfrak{p}_{j}\right)$.

THEOREM. There are $S$-units $\pi_{1}, \ldots, \pi_{\&-1}$ satisfying

$$
h\left(\pi_{1}\right) \cdots h\left(\pi_{\bullet-1}\right)<s !(c|D| \log P)^{s},
$$

where $c$ is a field constant

$$
c=\left(6 n^{3} / \log n\right)^{n},
$$

so that each $\alpha \in U_{S}$ can be written as a product

$$
\alpha=\rho \pi_{1}^{k_{1}} \cdots \pi_{s-1}^{k_{s-1}},
$$

with $\rho$ a root of unity and the rational integers $k_{i}$ satisfying

$$
\max _{1 \leqslant i \leqslant i-1}\left|k_{i}\right| \leqslant(s !)^{2} c^{2 s}(|D| \log P)^{\prime} h(\alpha) .
$$

The proof relies on a simple argument from the geometry of numbers and a result of Loxton and van der Poorten [5] on multiplicative relations in number fields.

\section{Preliminary Results}

A real-valued function $f$ on $\mathbf{R}^{m}$ is said to be a convex distance function if it satisfies

$$
\begin{aligned}
& f(\mathbf{x}) \geqslant 0 \text { for all } \mathbf{x} \in \mathbf{R}^{m}, \\
& f(\lambda \mathbf{x})=|\lambda| f(\mathbf{x}) \text { for all } \lambda \in \mathbf{R} \text { and } \mathbf{x} \in \mathbf{R}^{m}, \\
& f(\mathbf{x}+\mathbf{y}) \leqslant f(\mathbf{x})+f(\mathbf{y}) \text { for all } \mathbf{x}, \mathbf{y} \in \mathbf{R}^{\mathbf{m}} .
\end{aligned}
$$

LEMMA 1. Let $L$ be a full lattice in $\mathbf{R}^{m}$ and let $f$ be a convex distance function on $\mathbf{R}^{m}$. Suppose $x_{1}, \ldots, \mathbf{x}_{m}$ are linearly independent elements of $L$. Then there is a basis $\left\{b_{1}, \ldots, b_{m}\right\}$ for $L$ so that for $i=1, \ldots, m$

$$
f\left(\mathbf{b}_{i}\right) \leqslant \max \left(f\left(\mathbf{x}_{i}\right), \frac{1}{2}\left(f\left(\mathbf{x}_{1}\right)+\cdots+f\left(\mathbf{x}_{i}\right)\right)\right) .
$$

Proof: See, for example, Lemma 8, p.235 of Cassels [1]. 
REMARK. Since we may assume, without loss of generality, that $f\left(x_{1}\right) \leqslant \cdots \leqslant f\left(x_{m}\right)$, we obtain for $i=1, \ldots, m$ that

$$
f\left(\mathbf{b}_{i}\right) \leqslant \max \left(1, \frac{1}{2} i\right) f\left(\mathbf{x}_{i}\right)
$$

Denote by $\omega$ the number of roots of unity in $K$. If $\phi$ is Euler's totient function then $\phi(\omega) \mid[K: Q]$ and it follows that $\omega(K)<4 n \log \log 6 n$. Further let $\lambda(n)$ be a positive number with the property that $h(\alpha)<\lambda(n) / n$ and $\alpha$ have degree at most $n$; then $\alpha$ is zero or a root of unity. Then, from a result of Dobrowolski [2], it follows readily that we may choose $\lambda(n)$ as $\log n / 6 n^{3}$.

LEMMA 2. Let $\alpha_{1}, \ldots, \alpha_{k}$ be nonzero elements of $K$ with the property that there are rational integers $m_{1}, \ldots, m_{k}$ not all zero, so that

$$
\alpha_{1}^{m_{1}} \cdots \alpha_{k}^{m_{k}}=1
$$

Then there are rational integers $q_{1}, \ldots, q_{k}$, not all zero such that

and

$$
\begin{gathered}
\alpha_{1}^{q_{1}} \cdots \alpha_{k}^{q_{k}}=1 \\
\left|q_{1}\right| \leqslant(k-1) ! \omega \prod_{j \neq l}\left(n h\left(\alpha_{j}\right) / \lambda(n)\right) \text { for } l=1, \ldots, k
\end{gathered}
$$

Proof: See Loxton and van der Poorten [5].

\section{PROOF OF THE THEOREM}

Given $\alpha \in U_{S}$, denote by $\mathbf{v}(\alpha)$ the $s$-tuple $\left(\log |\alpha|_{v}\right)_{v \in S}$. This yields a correspondence between $U_{S}$ and a full lattice in $R^{-1}$, with just the roots of unity in $K$ corresponding to the zero vector.

Now, for each $i=1, \ldots, i=t$ let $\vartheta_{i}$ be the generator of the principal ideal $p_{i}^{h}$ satisfying

$$
\left.|\log | \operatorname{Norm} \vartheta_{i}\right|^{-1 / n}\left|\vartheta_{i}^{(j)}\right| \mid \leq \frac{1}{2} \operatorname{cr} R \text { for } j=1, \ldots, j=n,
$$

where the $\vartheta_{i}^{(j)}$ denote the field conjugates over $Q$ of $\vartheta_{i}$. Further, let $\left\{\varepsilon_{1}, \ldots, \varepsilon_{r}\right\}$ denote a multiplicatively independent set of (ordinary) units of $K$ satisfying

$$
h\left(\varepsilon_{1}\right) \cdots h\left(\varepsilon_{r}\right) \leqslant n^{n} R .
$$

For the propriety of the claims inherent in these definitions, see [4]. 
Given an s-tuple $x=\left(x_{1}, \ldots, x_{s}\right)$ we set $f(x)=(1 / 2 n)\left(\left|x_{1}\right|+\cdots+\left|x_{a}\right|\right)$. Then $f$ is a convex distance function and, for $\alpha \in S$, the product formula in $K$ yields

$$
\begin{aligned}
f(v(\alpha)) & =\left.\frac{1}{2 n} \sum_{v \in S}|\log | \alpha\right|_{v} \mid=\frac{1}{n} \sum_{v \in S} \max \left(0, \log |\alpha|_{v}\right) \\
& =\frac{1}{2 n} \sum_{v} \max \left(0, \log |\alpha|_{v}\right)=h(\alpha) .
\end{aligned}
$$

The elements $\varepsilon_{1}, \ldots, \varepsilon_{r}, \vartheta_{1}, \ldots, \vartheta_{t}$ are multiplicatively independent, and $s-1=$ $r+t$, so their corresponding vectors are linearly independent. Then, by Lemma 1 , we see that there is a set $\left\{\pi_{1}, \ldots, \pi_{0-1}\right\}$ of generators of $U_{S}$ with

$$
\begin{aligned}
h\left(\pi_{1}\right) \cdots h\left(\pi_{s-1}\right) & =f\left(v\left(\pi_{1}\right)\right) \cdots f\left(v\left(\pi_{s-1}\right)\right) \\
& \leqslant 2^{-(\bullet-1)}(s-1) ! f\left(v\left(\varepsilon_{1}\right)\right) \cdots f\left(v\left(\varepsilon_{r}\right)\right) f\left(v\left(\vartheta_{1}\right)\right) \cdots f\left(v\left(\vartheta_{t}\right)\right) \\
& =2^{-(\lrcorner-1)}(s-1) !\left(\prod_{i=1}^{r} h\left(\varepsilon_{i}\right)\right)\left(\prod_{j=1}^{t} h\left(\vartheta_{j}\right)\right),
\end{aligned}
$$

which establishes the first part of the theorem.

But the relation

asserts that

$$
\begin{aligned}
& \alpha=\rho \pi_{1}^{k_{1}} \cdots \pi_{:-1}^{k_{\ell-1}} \\
& 1=\alpha^{-\omega} \pi_{1}^{\omega k_{1}} \cdots \pi_{;-1}^{\omega k_{-1}},
\end{aligned}
$$

so by Lemma 2 we have rational integers $q_{0}, q_{1}, \ldots, q_{s-1}$ not all zero, such that

and

$$
\begin{gathered}
1=\alpha^{q 0} \pi_{1}^{q_{1}} \cdots \pi_{s-1}^{q_{\Delta-1}} \\
\left|q_{i}\right| \leqslant(s-1) ! \omega \mathrm{h}(\alpha) \prod_{j \neq i}\left(n \mathrm{~h}\left(\pi_{j}\right) / \lambda(n)\right) .
\end{gathered}
$$

Of course $q_{0} \neq 0$ since the $\pi_{j}$ are multiplicatively independent.

Moreover, the equation

$$
1=\alpha^{-\omega q 0} \pi_{1}^{\omega k_{1} q 0} \cdots \pi_{a-1}^{\omega k_{s-1} q_{0}}=\alpha^{-\omega q_{0}} \pi_{1}^{-\omega q_{1}} \cdots \pi_{s-1}^{-\omega q_{s-1}}
$$

yields

$$
1=\pi_{1}^{\omega k_{1} q_{0}+\omega q_{1}} \ldots \pi_{s-1}^{\omega k_{s-1} q_{0}+\omega q_{s-1}} \text {, }
$$

whence

$$
k_{1} q_{0}+q_{1}=\cdots=k_{\bullet-1} q_{0}+q_{\mathrm{s}-1}=0 \text {. }
$$

Hence $\left|k_{i}\right| \leq q_{i}$ for $i=1, \ldots, s-1$ which completes the proof. 


\section{CONCLUDING REMARKS}

The usual regulator argument (for example [6], p.103), already alluded to, does not yield an upper bound for the $k_{j}$ because the elements $\pi_{1}, \ldots, \pi_{s-1}$ do not necessarily generate relatively prime ideals. Thus there does not seem to be an obvious way to use such $\mathfrak{p}$-adic relations as

$$
\operatorname{ord}_{\mathfrak{p}} \alpha=\sum_{i=1}^{\leq-1} \operatorname{ord}_{\mathfrak{p}} \pi_{i}
$$

\section{REFERENCES}

[1] J.W.S. Cassels, An introduction to diophantine approximation: Cambridge Tracts in Mathematics and Mathematical Physics 45 (Cambridge University Press, Cambridge, 1965).

[2] E. Dobrowolski, 'On a question of Lehmer and the number of irreducible factors of a polynomial', Acta Arith. 34, 391-401.

[3] J.-H. Evertse and Györy, 'Thue-Mahler equations with a small number of solutions', $J$. für Math. 392 (1989), 1-21.

[4] K. Györy, 'On the solutions of linear diophantine equations in algebraic integers of bounded norm', Ann. Univ. Sci. Budapest, Eötvös Sect. Math. 22/23, 225-233.

[5] J.H. Loxton and A.J. van der Poorten, 'Multiplicative dependence in number fields', Acta Arith. 42 (1983), 291-302.

[6] T.N. Shorey and R. Tijdeman, Exponential diophantine equations: Cambridge Tracts in Mathematics 87 (Cambridge University Press, Cambridge, 1986).

Mathematics Institute

Kossuth Lájos University

H - 4010 Debrecen Pf. 12

Hungary 